\title{
Identification of a Highly Expressed 3-Hydroxy-3-Methylglutaryl-CoA Reductase Gene in the Root Tissue of Taraxacum kok-saghyz
}

\author{
Grisel Ponciano, Grace Q. Chen* \\ US Department of Agriculture, Agricultural Research Service, Western Regional Research Center, Albany, USA \\ Email: ${ }^{*}$ grace.chen@ars.usda.gov
}

Received 8 October 2014; revised 7 November 2014; accepted 20 November 2014

Academic Editor: Manish Arha, Rensselaer Polytechnic Institute, USA.

Copyright (C) 2014 by authors and Scientific Research Publishing Inc.

This work is licensed under the Creative Commons Attribution International License (CC BY).

http://creativecommons.org/licenses/by/4.0/

(c) (†) Open Access

\begin{abstract}
Kazakh dandelion (Taraxacum kok-saghyz, Tk) is a rubber-producing plant currently being investigated as a source of natural rubber for industrial applications. Like many other isoprenoids, rubber is a downstream product of the mevalonate pathway. The 3-hydroxy-3-methylglutaryl-CoA reductase (HMGR) enzyme catalyzes the conversion of 3-hydroxy-3-methylglutaryl-CoA to mevalonic acid, a key regulatory step in the MVA pathway. Such regulated steps provide targets for increases in isoprenoid and rubber contents via genetic engineering to increase enzyme activities. In this study, we identify a TkHMGR1 gene that is highly expressed in the roots of Kazakh dandelion, the main tissue where rubber is synthesized and stored. This finding paves the way for further molecular and genetic studies of the TKHMGR1 gene, and its role in rubber biosynthesis in $T k$ and other rubber-producing plants.
\end{abstract}

\section{Keywords}

3-Hydroxy-3-Methylglutaryl-CoA Reductase, Taraxacum kok-saghyz, Root, Gene Expression, Quantitative Polymerase Chain Reaction

\section{Introduction}

The main source of natural rubber in the world is the rubber tree Hevea (Hevea brasiliensis) grown mainly in Southeast Asia [1] [2]. Other rubber-producing plants, such as Kazakh dandelion (formerly Russian dandelion)

"Corresponding author.

How to cite this paper: Ponciano, G. and Chen, G.Q. (2014) Identification of a Highly Expressed 3-Hydroxy-3-Methylglutaryl-CoA Reductase Gene in the Root Tissue of Taraxacum kok-saghyz. American Journal of Plant Sciences, 5, 3603-3608. http://dx.doi.org/10.4236/ajps.2014.524376 
(Taraxacum kok-saghyz, $T$ ) and Guayule (Parthenium argentatum) are currently being developed as alternative crop sources of natural rubber [3]-[6].

Biosynthesis of rubber starts with an allylic pyrophosphate initiator, such as farnesyl pyrophosphate (FPP), followed by progressive addition of isopentenyl pyrophosphate (IPP) molecules [7]-[9]. The initiator and IPP molecules are synthesized by the mevalonate (MVA) pathway in the cytosol of plant cells [10] [11]. IPP is also synthesized in plastids via the 2-C-methyl-D-erythritol-4-phosphate (MEP) pathway [8] [11] [12]. The 3-hydroxy-3-methylglutaryl-CoA reductase (HMGR) is a key regulatory enzyme in the MVA pathway [13]-[16]. In all plants studied so far, HMGR is encoded by multiple genes, ranging from two in Arabidopsis to eight in cotton [17]. The different plant isoforms are commonly regulated at the transcriptional level [11] [18] [19]. Only a few cases of translational [20] and post-translational [21] [22] regulation have been reported. Among rubber producing plants, three HMGR isoforms have been cloned from Hevea, with isoform HbHMGR1 (NCBI Genbank accession number: X54659) associated with rubber biosynthesis [23] [24]. In Taraxacum brevicorniculatum, three HMGR isoforms have been characterized, each with distinct tissue expression profiles. Among them, TbHMGR 1 is the only isoform expressed in latex (the milky cytoplasm of the highly differentiated cells called laticifers) where rubber is synthesized [25]. Recently, one TkHMGR (HQ857601) sequence was released in GenBank, but it was not characterized.

Constitutive over expression of HMGR genes has proven to be effective in increasing production of selected isoprenoids and end-product sterols in plants [26]-[28]. The main organ of rubber synthesis and storage in $T k$ is the root. To increase rubber biosynthesis in $T k$ by means of genetic engineering, one approach is to over express a HMGR in the root tissue. Here we report the identification of a root-specific TkHMGR1 through data mining and gene expression studies.

\section{Materials and Methods}

\subsection{Plant Material}

Tk seeds were obtained from the United States Department of Agriculture-Agricultural Research Service National Plant Germplasm System, where they were deposited after collection in Kazakhstan in 2008 (accession No. KAZ08-014, ID W6-35169). Plants were germinated and grown in a greenhouse at temperatures between $28^{\circ} \mathrm{C}$ (day) and $18^{\circ} \mathrm{C}$ (night), with supplemental metal halide lighting to provide a 15-h-day length (1000 to 1250 $\left.\mu \mathrm{mol} \mathrm{m}{ }^{-2} \cdot \mathrm{s}^{-1}\right)$.

\subsection{RNA Extraction and Quantitative PCR Analysis}

Total RNA from root, leaf and flower tissues of greenhouse-grown $T k$ plants was extracted using a PowerPlant ${ }^{\circledR}$ RNA Isolation Kit with DNase (MoBio Laboratories Inc., USA). The total RNA was converted to cDNA using the Superscript III First-Strand Synthesis System for Reverse Transcriptase (RT)-Polymerase Chain Reaction (PCR) (Life Technologies, USA). Quantitative PCR (qPCR) on cDNAs of TkHMGR and 18S rRNA was performed using Applied Biosystems 7500 Fast Real Time PCR System. The following combinations of forward/ reverse primers were used for the qPCR reactions:

for TkHMGR1 5'-CTGGAACTATTGCTCCGACAA-3'/5'-GCCATGGCGGACCCTGTCAGG-3' and for Tk18S rRNA 5'-CGGCTACCACATCCAAGGAA-3'/5'-TGTCACTACCTCCCCGTGTCA-3'.

Other primer sets used in spatial and temporal analysis of $T k H M G R$-like sequences are:

TkHMGR2 5'-TTTGAAATTCTTCTTGGAGGAA-3'/5'-ACCATGTTCATCCCCATTGCATC-3';

TkHMGR3 5'-TTGCCACGGGACAAGACCCCGCT-3'/5'-GACTGTGAAGCTAGTTGAGTTCC-3';

TkHMGR4 5'-CGGTCAGACCCTGCCCGGAATGTG-3'/5'-TCTATGGAAGGCATGGTGACAGA-3';

TkHMGR5 5'-TTGCAGCACCGGTGATGCAATGGG-3'/5'-ACAGACTTTCCACGCCCTTCTATCCA-3'.

PCR amplification reactions were carried out in a volume of $25 \mu \mathrm{L}$ containing $20 \mathrm{ng}$ of cDNA, $0.5 \mu \mathrm{M}$ each of forward and reverse primers, and 1x SYBR ${ }^{\circledR}$ Green Master Mix (Applied Biosystems USA), using the following temperature regime: $95^{\circ} \mathrm{C}$ for $20 \mathrm{~s}$, followed by 40 cycles of $95^{\circ} \mathrm{C}$ for $3 \mathrm{~s}$ and $60^{\circ} \mathrm{C}$ for $30 \mathrm{~s}$. The relative copy numbers of a gene were averaged over triplicates in the same 96-well micro chamber plate. Expression of $T k H M G R$ was normalized to the expression of the constitutively expressed endogenous reference gene $18 S$ rRNA. The method of Pfaffl (2001) [29] was applied to calculate comparative expression levels between samples. The qPCR experiments were repeated at least twice. 


\section{Results and Discussion}

\subsection{Computational Analysis of TkHMGR-Like Expressed Sequences}

Hevea HMGR1 (X54659) was used as query for homology searches (tblastn) of a Tk root tissue expression library DNA sequences available at the National Center for Biotechnology Information (NCBI). Seventeen TkHMGR-like partial sequences were found with an average length of $750 \mathrm{bp}$ (Table 1). Eleven of the sequences were homologous to the N-terminus of Hevea HMGR1, three to the C-terminus, and three to the middle portion of the protein (between amino acids 167 - 426). The large numbers of $\mathrm{N}$-terminus homology sequences and the low representation of sequences with C-terminus homology suggest a technical problem during construction of the cDNA library. HMGR is known to be present in plants in several isoforms. Without full-length TkHMGR sequence information, it is impossible to determine the number of TkHMGR isoforms. However, spatial (organ specific) and temporal (age specific) expression analysis of these sequences can provide useful information about the presence of tissue-specific isoforms.

\subsection{Spatial and Temporal Analysis of Tk HMGR-Like Sequences}

We aligned all 17 Tk sequences against the Hevea HMGR1 gene (X54659) and designed five sets of PCR primers. Computational analysis of the five primer sets predicted that each would amplify at least one of the following 6 sequences, GO661008, GO662289, GO668051, GO666861, GO665221, and GO670200. PCR amplifications were performed on cDNAs derived from total RNA extracted from leaf, root, and flower tissues of 11 weeks old $T k$ plants. All primers sets amplified a product in all tissues (data not shown). However one primer set (Materials and Methods) consistently amplified an abundant product from cDNAs from root tissues. This primer set, herein called TkHMGR1 primers, was used for quantitative expression analysis. Computational analysis of TkHMGR1 primers predicted only one PCR product from only one sequence, GO666861, and, interestingly, no amplification from the cloned TkHMGR sequence (HQ857601).

Table 1. List of TkHMGR-like sequences showing homology to Hevea HMGR1.

\begin{tabular}{|c|c|c|c|c|}
\hline Accession No. & \% Coverage* & Homology location** & \% Identity & Length (bases) \\
\hline GO661008 & 42 & Center & 85 & 768 \\
\hline GO662289 & 40 & Center & 83 & 760 \\
\hline GO668051 & 44 & Center & 85 & 773 \\
\hline GO661429 & 45 & N-term & 67 & 783 \\
\hline GO666861 & 33 & C-term & 89 & 655 \\
\hline GO661610 & 36 & N-term & 60 & 786 \\
\hline GO660958 & 38 & N-term & 57 & 750 \\
\hline GO661845 & 37 & N-term & 58 & 807 \\
\hline GO661812 & 35 & N-term & 59 & 775 \\
\hline GO662014 & 34 & $\mathrm{~N}$-term & 58 & 752 \\
\hline GO665221 & 21 & C-term & 85 & 671 \\
\hline GO661926 & 36 & N-term & 52 & 762 \\
\hline GO670200 & 19 & C-term & 88 & 665 \\
\hline GO660861 & 34 & N-term & 53 & 754 \\
\hline GO662370 & 34 & N-term & 53 & 781 \\
\hline GO661179 & 33 & N-term & 53 & 758 \\
\hline GO662172 & 32 & N-term & 52 & 725 \\
\hline
\end{tabular}

*Compared to Hevea HMGR1 sequence (X54659); **Location in Hevea HMGR1 sequence (X54659). 
To examine the relationship between rubber accumulation and TkHMGR1 expression, we further quantified TkHMGR1 transcript levels in root and leaf of young ( $\sim 11$ weeks old) and mature ( 20 weeks old) Tk plants using qPCR technology. In our lab, rubber extractions from $T k$ roots typically yield $4 \mathrm{mg} / \mathrm{g}$ fresh weight from 12-week-old plants and $10 \mathrm{mg} / \mathrm{g}$ fresh weight from 20-week old plants). No rubber was detected in leaf tissues. As shown in Figure 1, we detected 11-fold or 3-fold higher of TkHMGR1 transcript levels in roots than in leaves from young or mature plants, respectively. The results indicated that the expression of TkHMGR1 was higher in root than leaf during the active growth phase of $T k$ plants. Although functional studies need to be carried out in order to determine the enzymatic activity and substrate specificity of TkHMGR1 in rubber metabolism, it is likely that $T k H M G R 1$ contributes to rubber synthesis in $T k$ roots based on its expression profile.

Rubber is the end metabolite of the MVA biosynthesis pathway. The amount of rubber in mature root is the result of continuous synthesis and accumulation over the lifespan of the plant. Although we observed a decline in expression of TkHMGR1 in roots when plants became mature (Figure 1), the level of the transcript was probably still high enough to produce active TkHMGR1 enzymes for rubber biosynthesis. It is also possible that TkHMGR1 is a relatively stable protein, and thus supports continuous biosynthesis of rubber over development, leading to the high accumulation of rubber in root.

In summary, we have identified the TkHMGR1 isoform that is highly expressed in $T k$ root. The C-terminal part of TkHMGR1 sequences are represented by GenBank accession GO666861. The partial sequence provided the basis for the design of isoform-specific primers that can be used to isolate full-length TkHMGR1 and eventually its promoter sequences. The likely role that TkHMGR1 plays in rubber synthesis suggests that its overexpression in guayule or $T k$ could increase rubber production. In addition, the TkHMGR promoter can be used to drive gene expression for other key enzymes/proteins associated with rubber synthesis in $T k$ root, such as the genes for IPP synthase, cis-prenyltransferase, rubber elongation factor, etc. Thus, the sequence identified in this study could lead to other valuable tools for genetic engineering of rubber-producing crops.

\section{Acknowledgements}

This study was conducted with support from the US Department of Agriculture-Agricultural Research Service-Current Research Information System Project 5325-21410-020-00D. The authors wish to thank Ann Blechl and Colleen McMahan for critical reading of the manuscript, and Kumiko Johnson and Eva Morales for assisting in plant care in the greenhouse. Thanks are extended to Barbara Hellier for kindly supplying the $T k$ seeds. USDA is an equal opportunity provider and employer. Mention of a specific product name by the United States

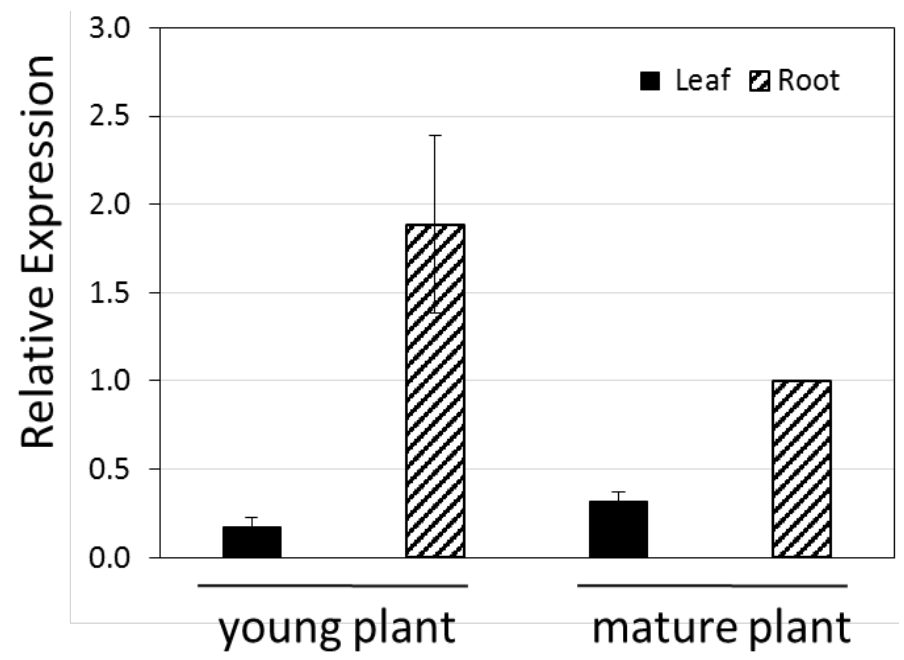

Figure 1. Expression of TkHMGR1 in root and leaf of young (11 week) and mature (20 week) $T k$ greenhouse plants. Expression levels determined by qPCR are relative to those of $18 S$ ribosomal RNA in the same samples. Each data point represents the mean \pm SD of three replicates. 
Department of Agriculture does not constitute an endorsement and does not imply a recommendation over other suitable products.

\section{References}

[1] Rivano, F., Mattos, C., Cardoso, S., Martinez, M., Ceballos, V., Le Guen, V. and Garcia, D. (2013) Breeding Hevea brasiliensis for Yield, Growth and SALB Resistance for High Disease Environments. Industrial Crops and Products, 44, 659-670. http://dx.doi.org/10.1016/j.indcrop.2012.09.005

[2] Fox, J., Castella, J., Ziegler, A. and Westley, S. (2014) Rubber Plantations Expand in Mountainous Southeast Asia: What Are the Consequences for the Environment? Analysis from the East West Center, 114, 1-8.

[3] Van Beilen, J. and Poirier, Y. (2007) Establishment of New Crops for the Production of Natural Rubber. Trends in Biotechnology, 25, 522-529. http://dx.doi.org/10.1016/j.tibtech.2007.08.009

[4] Canter, N. (2011) Natural Rubber from Dandelions: A Russian Flower May Hold the Answer to a Worldwide Shortage. Tribology and Lubrication Technology, 67, 14-15.

[5] Hellier, B. (2011) Collecting in Central Asia and the Caucasus: US National Plant Germplasm System Plant Explorations. HortScience, 46, 1438-1439.

[6] Sfeir, N., Chapuset, T., Palu, S., Lancon, F., Amor, A., Garcia, J. and Snoeck, D. (2014) Technical and Economic Feasibility of a Guayule Commodity Chain in Mediterranean Europe. Industrial Crops and Products, 59, 55-62. http://dx.doi.org/10.1016/j.indcrop.2014.04.043

[7] Lynen, F. and Henning, U. (1960) Biological Path to Natural Rubber. Angewandte Chemie, 72.

[8] Cornish, K. (2001) Similarities and Differences in Rubber Biochemistry among Plant Species. Phytochemistry, 57, 1123-1134. http://dx.doi.org/10.1016/S0031-9422(01)00097-8

[9] Tanaka, Y. (2001) Structural Characterization of Natural Polyisoprenes: Solve the Mystery of Natural Rubber Based on Structural Study. Rubber Chemistry and Technology, 74, 355-375. http://dx.doi.org/10.5254/1.3547643

[10] Chappell, J. (1995) Biochemistry and Molecular Biology of the Isoprenoid Biosynthetic Pathway in Plants. Annual Review of Plant Physiology and Plant Molecular Biology, 46, 521-547.

http://dx.doi.org/10.1146/annurev.pp.46.060195.002513

[11] Vranova, E., Coman, D. and Gruissem, W. (2013) Network Analysis of the MVA and MEP Pathways for Isoprenoid Synthesis. Annual Review of Plant Biology, 64, 665-700. http://dx.doi.org/10.1146/annurev-arplant-050312-120116

[12] Sando, T., Takeno, S., Watanabe, N., Okumoto, H., Kuzuyama, T., Yamashita, A., Hattori, M., Ogasawara, N., Fukusaki, E. and Kobayashi, A. (2008) Cloning and Characterization of the 2-C-methyl-D-erythritol-4-phosphate (MEP) Pathway Genes of the Natural-Rubber Producing Plant, Hevea brasiliensis. Bioscience, Biotechnology and Biochemistry, 72, 2903-2917. http://dx.doi.org/10.1271/bbb.80387

[13] Bach, T. (1986) Hydroxymethylglutaryl-CoA Reductase, A Key Enzyme in Phytosterol Synthesis? Lipids, 21, 82-88. http://dx.doi.org/10.1007/BF02534307

[14] Goldstein, J. and Brown, M. (1990) Regulation of the Mevalonate Pathway. Nature, 343, 425-430. http://dx.doi.org/10.1038/343425a0

[15] Hampton, R., Dimster-Denk, D. and Rine, J. (1996) The Biology of HMG-CoA Reductase; the Pros of Contra-Regulation. Trends in Biological Science, 21, 140-145. http://dx.doi.org/10.1016/S0968-0004(96)80168-X

[16] Espenshade, P. and Hughes, A. (2007) Regulation of Sterol Synthesis in Eukaryotes. Annual Review of Genetics, 41, 401-427. http://dx.doi.org/10.1146/annurev.genet.41.110306.130315

[17] Li, W., Liu, W., Wei, H., He, Q., Chen, J., Zhang, B. and Zhu, S. (2014) Species-Specific Expansion of Molecular Evolution of the 3-Hydroxy-3-Methylglutaryl Coenzyme A Reductase (HMGR) Gene Family in Plants. PloS ONE, 9, e94172. http://dx.doi.org/10.1371/journal.pone.0094172

[18] Lumbreras, V., Campos, N. and Boronat, A.(1995) The Use of An Alternative Promoter in the Arabidopsis thaliana HMG1 Gene Generates an Mrna that Encodes a Novel 3-Hydroxy-3-Methylglutaryl Coenzyme A Reductase Isoform with an Extended N-Terminal Region. The Plant Journal, 8, 541-549.

http://dx.doi.org/10.1046/j.1365-313X.1995.8040541.X

[19] Kondo, K., Uritani, I. and Oba, K. (2003) Induction Mechanism of 3-Hydroxy-3-Methylglutaryl-Coa Reductase in Potato Tuber and Sweet Potato Root Tissues. Biosciences, Biotechnology and Biochemistry, 67, 1007-1017. http://dx.doi.org/10.1271/bbb.67.1007

[20] Yoshioka, A., Miho, M., Yukie, H. and Noriyuki, D. (1996) Expression of Genes for Phenylalanine Ammonia-Lyase and 3-Hydroxy-3-Methylglutaryl CoA Reductase in Aged Potato Tubers Infected with Phytophthora infestans. Plant Cell Physiology, 37, 81-90. http://dx.doi.org/10.1093/oxfordjournals.pcp.a028917 
[21] Dale, S., Arro, M., Becerra, B., Morrice, N., Boronat, A., Hardie, D. and Ferrer, A. (1995) Bacterial Expression of the Catalytic Domain of 3-Hydroxy-3-Methylglutaryl-CoA Reductase (Isoform HMGR1) from Arabidopsis thaliana, and Its Inactivation by Phosphorylation at Ser577 by Brassica oleracea 3-Hydroxy-3-Methylglutaryl-CoA Reductase Kinase. European Journal of Biochemistry, 233, 506-513. http://dx.doi.org/10.1111/j.1432-1033.1995.506_2.x

[22] Leivar, P., Antolin-Llovera, M., Ferrero, S., Closa, M., Arro, M., Ferrer, A., Boronat, A. and Campos, N. (2011) Multilevel Control of Arabidopsis 3-Hydroxy-3-Methylglutaryl Coenzyme A Reductase by Protein Phosphatase 2A. The Plant Cell, 23, 1494-1511.

[23] Chye, M., Kush, A., Tan, C. and Chua, N. (1991) Characterization of cDNA and Genomic Clones Encoding 3-Hydroxy-3-Methylglutaryl-Coenzyme A Reductase from Hevea brasiliensis. Plant Molecular Biology, 16, 567-577. http://dx.doi.org/10.1007/BF00023422

[24] Chye, M., Tan, C. and Chua, N. (1992) Three Genes Enconde 3-Hydroxy-3-Methylglutaryl-Coenzyme A Reductase in Hevea brasiliensis: hmg1 and hmg3 Are Differentially Expressed. Plant Molecular Biology, 19, 473-484. http://dx.doi.org/10.1007/BF00023395

[25] van Deene, N., Bachmann, A., Schmidt, T., Schaller, H., Sand, J., Prufer, D. and Gronover, C. (2012) Molecular Cloning of Mevalonate Pathway Genes from Taraxacum brevicorniculatum and Functional Characterization of the Key Enzyme 3-Hydroxy-3-Methylglutaryl-Coenzyme A Reductase. Molecular Biology Reports, 39, 4337-4349. http://dx.doi.org/10.1007/s11033-011-1221-4

[26] Holmberg, N., Harker, M., Wallace, A., Cayton, J., Gibbard, C. and Safford, R. (2003) Co-Expression of N-Terminal Truncated 3-Hydroxy-3-Methylglutaryl CoA Reductase and C24-Sterol Methyltransferase Type 1 in Transgenic Tobacco Enhances Carbon Flux Towards End-Product Sterols. The Plant Journal, 36, 12-20. http://dx.doi.org/10.1046/j.1365-313X.2003.01851.x

[27] Ro, D., Paradise, E., Ouellet, M., Fisher, K., Newman, K., Ndungu, J., Ho, K., Eachus, R., Ham, T., Kirby, J., Chang, M., Withers, S., Shiba, Y., Sarpong, R. and Keasling, J. (2006) Production of the Antimalarial Drug Precursor Artemisinic Acid in Engineered Yeast. Nature, 440, 940-943. http://dx.doi.org/10.1038/nature04640

[28] Kim, Y., Lee, O., Oh, J., Jang, M. and Yang, D. (2014) Functional Analysis of 3-Hydroxy-3-Methylglutaryl Coenzyme A Reductase Encoding Genes in Triterpene Saponin-Producing Ginseng. Plant Physiology, 165, 373-387. http://dx.doi.org/10.1104/pp.113.222596

[29] Pfaffl, M.W. (2001) A New Mathematical Model for Relative Quantification in Real-Time RT-PCR. Nucleic Acids Research, 29, e45. http://dx.doi.org/10.1093/nar/29.9.e45 
Scientific Research Publishing (SCIRP) is one of the largest Open Access journal publishers. It is currently publishing more than 200 open access, online, peer-reviewed journals covering a wide range of academic disciplines. SCIRP serves the worldwide academic communities and contributes to the progress and application of science with its publication.

Other selected journals from SCIRP are listed as below. Submit your manuscript to us via either submit@scirp.org or Online Submission Portal.
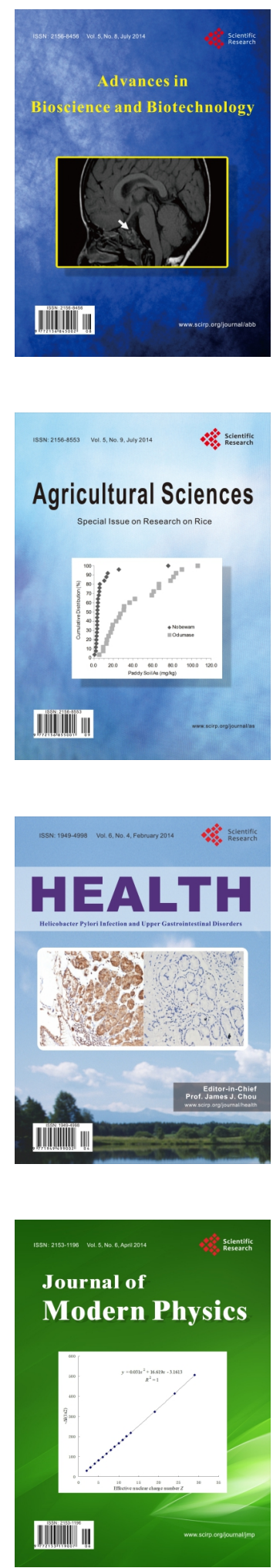
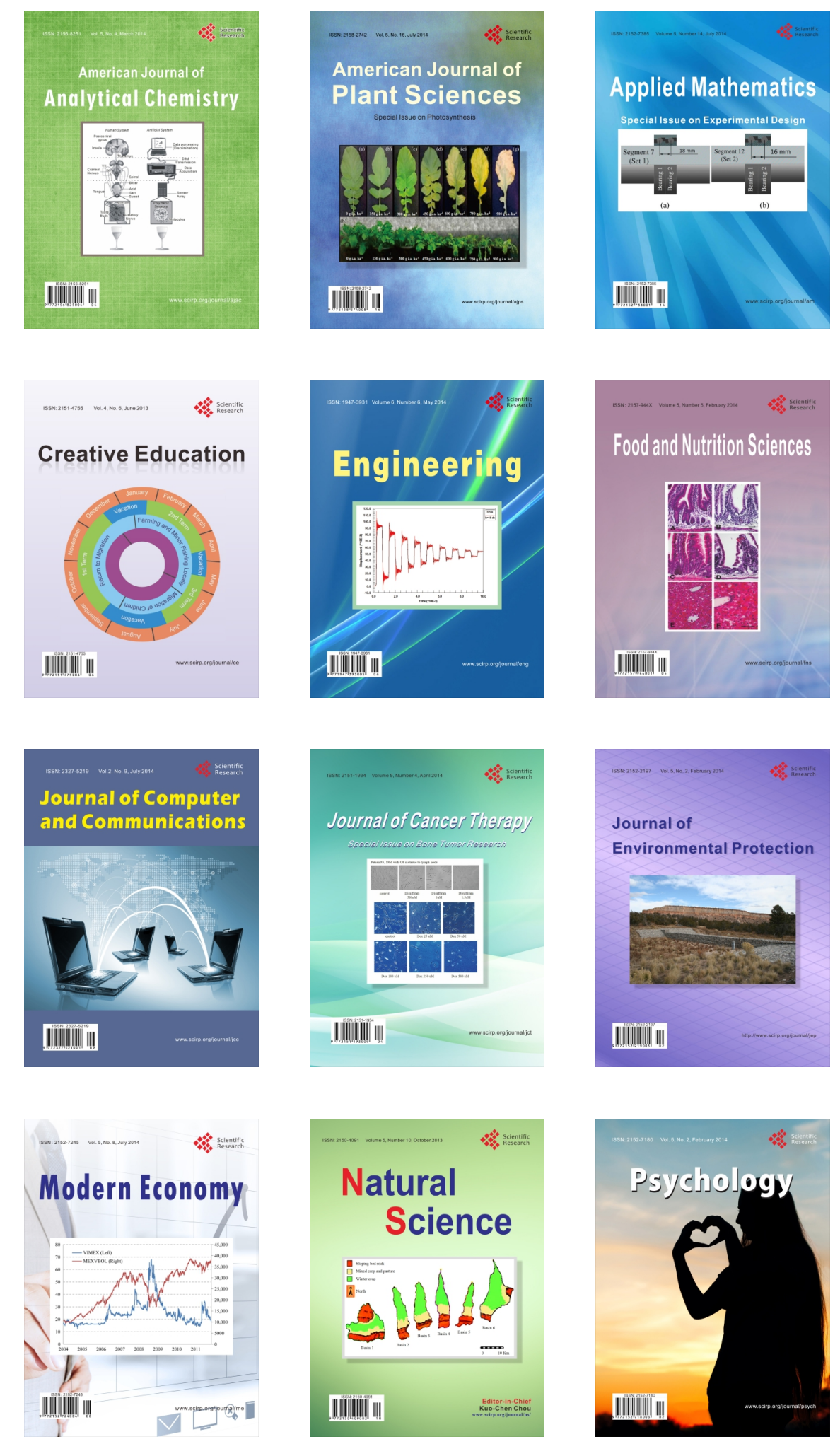